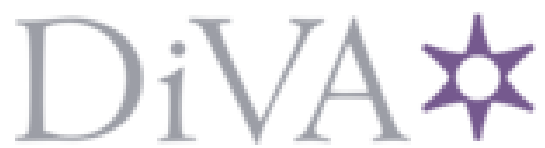

http://www.diva-portal.org

Preprint

This is the submitted version of a paper presented at 8th IEEE International Workshop on Information Forensics and Security, WIFS 2016, NYUAbu Dhabi, United Arab Emirates, 4 December 2016 through 7 December 2016.

Citation for the original published paper:

Vu, M T., Oechtering, T J., Skoglund, M. (2017)

Polar code for secure Wyner-Ziv coding

In: 8th IEEE International Workshop on Information Forensics and Security, WIFS

2016, 7823897 Institute of Electrical and Electronics Engineers (IEEE)

https://doi.org/10.1109/WIFS.2016.7823897

N.B. When citing this work, cite the original published paper.

The work was supported by the Swedish Research Council (VR) project under Grant 2016-03853.

Permanent link to this version:

http://urn.kb.se/resolve?urn=urn:nbn:se:kth:diva-208455 


\title{
Polar code for secure Wyner-Ziv coding
}

\author{
Minh Thanh Vu, Tobias J. Oechtering and Mikael Skoglund \\ School of Electrical Engineering \\ KTH Royal Institute of Technology
}

\begin{abstract}
Secure source coding provides the methods to compress data efficiently taking the risks of unauthorized access into account. However standard information-theoretic achievability proofs only show the existence of a coding scheme without providing a concrete code design. In the paper we provide an alternative proof of achievability for the secure Wyner-Ziv source coding problem with side information at the eavesdropper using polar coding. Due to the recent algorithmic advances in the design of polar codes, our result is highly relevant for future big data applications since it provides the basis for further developments.
\end{abstract}

\section{INTRODUCTION}

Secrecy has become an eminent theme in academia and practice due to major advances in technologies. The notion of information theoretic secrecy was developed by Shannon in his work for the cipher system. Shannon's perfect secrecy criterion was relaxed to weak secrecy later by Wyner's work for the wiretap channel. Therein the secrecy is measured by the equivocation of the transmitted message given the channel output at the eavesdropper. Recently weak secrecy has been used to measure the strength of the network against adversaries in source coding settings with side information in [1], [2]. In these works, the secrecy is measured by the equivocation of the source given transmitted information and the side information at the eavesdropper. Since the equivocation constraint limits the amount of information that is leaked to the eavesdropper, it characterizes, in a sense, the best attainable effort at the eavesdropper.

Along another line, channel polarization, the main component of polar codes for binary input symmetric channels, was studied by Arikan in [3]. Notable characteristics of polar codes are low-scaling decoding $(\mathcal{O}(n \log n))$, and provable performance in the asymptotic regime. Generalization of channel polarization to Galois fields and arbitrary $q$-ary alphabets are carried out in [4] and [5], respectively. Furthermore, channel polarization has been generalized to source polarization in [6], which was used to develop coding schemes for lossless source coding with side information [6], broadcast channels [7], asymmetric channels and lossy source coding [8]. In parallel with the development of polar codes, their applications to secrecy problems have been also studied in [9], [10] for wiretap channels, [11] for bidirectional broadcast channels with confidential messages and [12] for Yamamoto's secure lossy source coding.

Future big data applications stimulate the development of source compression codes for efficiently storing data. Moreover, recent achievements show that polar codes are capable of outperforming state-of-the-art coding with acceptable complexity [13]. These facts motivate us to study polar coding schemes for the secure source coding problem. In this work we consider the Wyner-Ziv network [14] in the presence of an eavesdropper with its own side information. The complete characterization of the setting is given in [2], in which the achievalibity is proved using typical coding arguments. We propose an alternative approach using polar coding in this work. In contrast to typical coding where one needs to search through an exponential number of codewords, polar coding is a linear coding scheme, which offers significant advantages in terms of encoding complexity. Our work, hence, brings secure lossy source coding one step further to practice.

The paper is organized as follows. In Section IIProblem Statementsection. 2 we recall the problem formulation and the previous result on the rate-distortion-equivocation region using typical coding. We then state our result. The proof of the result is given in Section IIIProofsection.3. In Section IVConclusionsection. 4 we summarize the paper.

\section{Problem Statement}

We begin by introducing some notations. Random variables, their realizations and their alphabets are denoted by upper cases, lower cases, and calligraphic letters, respectively. We consider only finite alphabets in our work. Some probability distributions are denoted by blackboard bold letters. We use $\mathbb{E}_{\mathbb{Q}}\{\cdot\}$ to denote the expectation with respect to the underlying distribution $\mathbb{Q}$. Similarly, we also use notations $H_{\mathbb{Q}}(\cdot)$ and $I_{\mathbb{Q}}(\cdot ; \cdot)$ to denote the entropy and the mutual information with respect to the distribution $\mathbb{Q}$. The cardinality of a set $\mathcal{A}$ is denoted by $|\mathcal{A}|$.

In our setting (cf. Fig. 2Partitioning the set $[1: n]$ into corresponding subsets. Note that the indices in each set can be arbitrarily.figure.caption.2), the source information $X^{n}$ is compressed and sent over the network, which is intercepted by an eavesdropper, subject to a distortion constraint at the destination. The destination and the eavesdropper have their own side information. The letter distortion measure at the decoder is given by $d: \mathcal{X} \times \hat{\mathcal{X}} \rightarrow\left[0, d_{\max }\right]$, where $\hat{\mathcal{X}}$ is the reconstruction alphabet. The sequence distortion of measure is defined, with abuse of notation, as

$$
d\left(x^{n}, \hat{x}^{n}\right)=\frac{1}{n} \sum_{i=1}^{n} d\left(x_{i}, \hat{x}_{i}\right) .
$$

The secrecy level of the coding scheme is measured by the equivocation $\frac{1}{n} H\left(X^{n} \mid M, E^{n}\right)$ where $M$ is the transmitted message. The equivocation describes the remaining average uncertainty about the source $X^{n}$ at the eavesdropper after intercepting the message $M$ given its own side information $E^{n}$. It also provides us an assessment of the system against the risks of being unauthorizedly accessed.

Definition 1. An $(n, \mathcal{M})$-code for secrecy is defined via a stochastic encoder

$$
\phi: \mathcal{X}^{n} \rightarrow \mathcal{M}
$$


and a decoding function

$$
\psi: \mathcal{M} \times \mathcal{Y}^{n} \rightarrow \hat{\mathcal{X}}^{n} .
$$

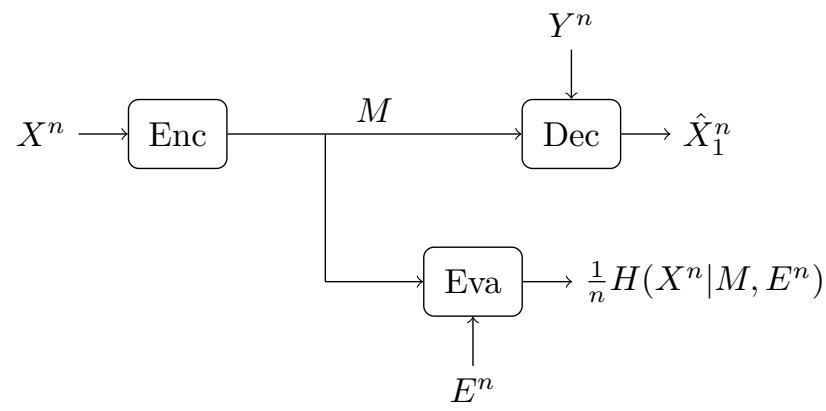

Fig. 1: Wyner-Ziv network in the presence of an eavesdropper equipped with its own side information.

Definition 2. A rate-distortion-equivocation tuple $(R, D, \Delta)$ is achievable if for every $\epsilon>0$ the following conditions are fulfilled

$$
\begin{aligned}
\frac{1}{n} \log |\mathcal{M}| & <R+\epsilon, \\
\mathbb{E}\left[d\left(X^{n}, \hat{X}^{n}\right)\right] & <D+\epsilon, \\
\frac{1}{n} H\left(X^{n} \mid M, E^{n}\right) & >\Delta-\epsilon,
\end{aligned}
$$

for all sufficiently large $n$. The set of all rate-distortionequivocation tuples is the rate-distortion-equivocation region.

In [2, Theorem 3] it is shown by joint typicality encoding and decoding that the rate-distortion-equivocation region is given by

$$
\begin{aligned}
& R \geq I(X ; V \mid Y), \\
& D \geq E[d(X, f(V, Y))], \\
& \Delta \leq H(X \mid V, Y)+I(X ; Y \mid U)-I(X ; E \mid U),
\end{aligned}
$$

where $U-V-X-(Y, E)$, and $f: \mathcal{V} \times \mathcal{Y} \rightarrow \hat{\mathcal{X}}$ is a deterministic reconstruction mapping. Note that due to the Markov chain the equivocation constraint is equivalent to

$$
\Delta \leq H(X \mid U, E)-I(X ; V \mid U, Y) .
$$

Our result is summarized in the following theorem.

Theorem 1. For every $\epsilon>0$ there exists a polar code such that rate-distortion-equivocation tuples satisfying (2Problem Statementequation.2.2) are achievable for all sufficiently large $n$ subject to an additional constraint that both $U$ and $V$ are binary.

We only show through the proof in Section IIIProofsection. 3 the existence of a set of frozen symbols such that the desired distortion and equivocation levels are guaranteed. In principle one can use the brute-force approach to search for a set of frozen symbols. An efficient algorithm for this task is not available at the moment.

Remark 1. In [2] the authors use a coding approach based on typical sets, in which the encoding function is deterministic, to show the achievability of rate-distortion-region
(2Problem Statementequation.2.2). As will be shown in Section IIIProofsection. 3 a stochastic encoder is employed in our proof.

Remark 2. In Theorem 1theorem. 2.1 we limit the alphabets of the auxiliary random variables $U$ and $V$ to be binary. Extensions to $q$-ary alphabets can be possible if general polarization theorems in [4], [5] are applied.

\section{PROOF}

Before proceeding to the proof of Theorem 1theorem.2.1 we recall some additional information as follows. For a joint pmf $p_{X Y}$ on the space $\mathcal{X} \times \mathcal{Y}$, where $\mathcal{X}$ is the binary alphabet $\{0,1\}$, the Bhattacharyya parameter is defined in [6] as

$$
Z(X \mid Y)=2 \sum_{y} p_{Y}(y) \sqrt{p_{X \mid Y}(0 \mid y) p_{X \mid Y}(1 \mid y)} .
$$

It has the following properties (cf. [6, Proposition 2])

$$
\begin{aligned}
(Z(X \mid Y))^{2} & \leq H(X \mid Y), \\
H(X \mid Y) & \leq \log _{2}(1+Z(X \mid Y)) .
\end{aligned}
$$

We note that since the Bhattacharyya parameter is in the interval $[0,1]$, we then have

$$
\log _{2}(1+Z(X \mid Y)) \leq \log _{2}\left(e^{Z(X \mid Y)}\right)=\log _{2}(e) Z(X \mid Y) .
$$

Let $G_{n}$ be the polar transformation matrix for binary inputs. Note that, in $\mathbb{F}_{2}, G_{n}^{-1}=G_{n}$. In [8, Theorem 1] the following property was proven.

Theorem 2. For any $0<\beta<1 / 2$, i.i.d. random variables $(X, Y)$, and $U^{n}=X^{n} G_{n}$,

$$
\begin{aligned}
& \lim _{n \rightarrow \infty} \frac{1}{n} \mid\left\{i: Z\left(U_{i} \mid U^{i-1}, Y^{n}\right) \leq 2^{-n^{\beta}},\right. \text { and } \\
& \left.Z\left(U_{i} \mid U^{i-1}\right) \geq 1-2^{-n^{\beta}}\right\} \mid=I(X ; Y), \\
& \lim _{n \rightarrow \infty} \frac{1}{n} \mid\left\{i: Z\left(U_{i} \mid U^{i-1}, Y^{n}\right) \geq 1-2^{-n^{\beta}},\right. \text { or } \\
& \left.Z\left(U_{i} \mid U^{i-1}\right) \leq 2^{-n^{\beta}}\right\} \mid=1-I(X ; Y) .
\end{aligned}
$$

We first provide an overview of the code design. Recall that we consider a block code of length $n$. Further, we use superposition coding as in [2] with two random variables $U, V$. Consider the first layer of information which is described by the random variable $U$. In Fig. 2Partitioning the set $[1: n]$ into corresponding subsets. Note that the indices in each set can be arbitrarily.figure.caption. 2 we illustrate the encoding and decoding process related to $U$. The set of indices $[1: n]$ is partitioned into different sets $\mathcal{A}_{U}, \mathcal{D}_{U}$, and $\mathcal{F}_{U}$ through polar transformation from $U^{n}$ to $\tilde{U}^{n}$, i.e., $\tilde{U}^{n}=U^{n} G_{n}$. The set $\mathcal{D}_{U}$ includes indices $i$ such that $\tilde{U}_{i}$ is almost deterministic given information contained in the sequence $\tilde{U}^{i-1}$, which implies that communicating this set is not necessary and we can recover the sequence $\tilde{U}_{\mathcal{D}_{U}}$ by a suitable function with high probability. The set $\mathcal{F}_{U}$ consists of indices $i$ such that $\tilde{U}_{i}$ is almost independent of the tuple $\left(\tilde{U}^{i-1}, X^{n}\right)$, which implies $\mathcal{F}_{U}$ does not provide much information about the source and also can not be reconstructed easily. Therefore, we fix the information in this set and share between the 
transmitter and the receiver. The symbols in $\mathcal{F}_{U}$ are the so called frozen symbols. Consequently, the source sequence is compressed mainly through the set $\mathcal{A}_{U}$. Due to the presence of the side information sequence $y^{n}$, we can recover a subset $\mathcal{B}_{U}$ of $\mathcal{A}_{U}$. In summary, the rate contributed by the first layer is determined through the cardinality of the set $\mathcal{A}_{U} \backslash \mathcal{B}_{U}$. A similar interpretation can be done for the second layer $V$.

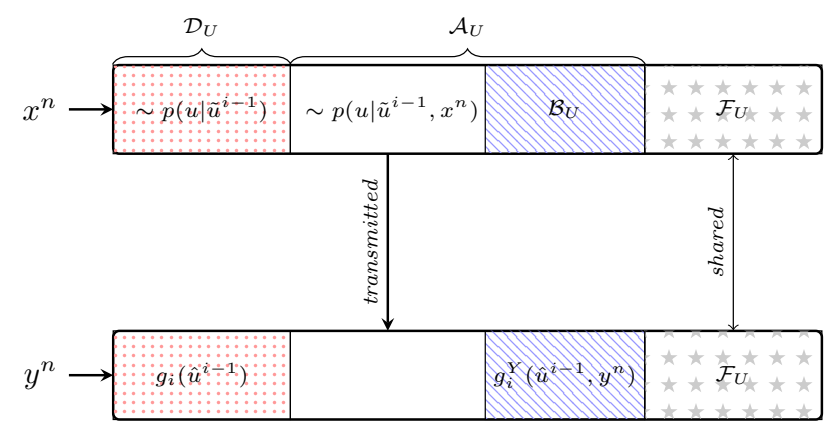

Fig. 2: Partitioning the set $[1: n]$ into corresponding subsets. Note that the indices in each set can be arbitrarily.

We now begin with our main proof.

Fix a conditional distribution $p_{U V \mid X}$ such that $U-V-X-$ $(Y E)$, i.e., the joint pmf $p_{X Y E U V}$ is given as

$$
p_{X Y E U V}=p_{U \mid V} p_{V \mid X} p_{X Y E}
$$

and a deterministic reconstruction mapping $f$ such that the following condition is satisfied

$$
\mathbb{E}[d(X, f(V, Y))] \leq D .
$$

Given a tuple $\left(u^{n}, v^{n}, x^{n}, y^{n}, e^{n}\right)$ generated i.i.d. from the joint distribution $p_{U V X Y E}$, we define the following pair of sequences

$$
\left[\begin{array}{c}
\tilde{u}^{n} \\
\tilde{v}^{n}
\end{array}\right]=\left[\begin{array}{l}
u^{n} \\
v^{n}
\end{array}\right] G_{n},
$$

where operations are carried out in $\mathbb{F}_{2}$. The transformation induces a probability distribution

$$
\mathbb{P}=p_{X^{n} Y^{n} E^{n}} \prod_{i=1}^{n} p\left(\tilde{u}_{i} \mid \tilde{u}^{i-1}, x^{n}\right) \prod_{i=1}^{n} p\left(\tilde{v}_{i} \mid \tilde{v}^{i-1}, \tilde{u}^{n}, x^{n}\right) .
$$

Note that arguments of $\mathbb{P}$ are implicitly defined. We observe that

$$
p\left(\tilde{v}_{i} \mid \tilde{v}^{i-1}, \tilde{u}^{n}, x^{n}\right)=p\left(\tilde{v}_{i} \mid \tilde{v}^{i-1}, u^{n}, x^{n}\right), \forall i \in[1: n],
$$

since $G_{n}$ is invertible. For simplicity, we denote $\delta_{n}=2^{-n^{\beta}}$, where $0<\beta<1 / 2$. Define $\mathcal{A}_{U}$ to be a subset of size $\left|\mathcal{A}_{U}\right|=$ $n R_{U}^{a}$ of $[1: n]$ such that

$$
Z\left(\tilde{U}_{i} \mid \tilde{U}^{i-1}, X^{n}\right) \geq 1-\delta_{n}, \text { or } Z\left(\tilde{U}_{i} \mid \tilde{U}^{i-1}\right) \leq \delta_{n},
$$

for all $i \in \mathcal{A}_{U}^{c}$. From Theorem 2theorem.3.2 such a set exists if $R_{U}^{a}>I(X ; U)$ for sufficiently large $n$. We observe that the set $A_{U}$ contains the set

$$
\left\{i: Z\left(\tilde{U}_{i} \mid \tilde{U}^{i-1}, X^{n}\right)<1-\delta_{n}, \text { and } Z\left(\tilde{U}_{i} \mid \tilde{U}^{i-1}\right)>\delta_{n}\right\} .
$$

Furthermore, let $\mathcal{B}_{U}$ be a subset of $[1: n]$ such that $\left|\mathcal{B}_{U}\right|=$ $n R_{U}^{b}$, and

$$
Z\left(\tilde{U}_{i} \mid \tilde{U}^{i-1}, Y^{n}\right) \leq \delta_{n} \text {, and } Z\left(\tilde{U}_{i} \mid \tilde{U}^{i-1}\right) \geq 1-\delta_{n}, \forall i \in \mathcal{B}_{U}
$$

Theorem 2theorem.3.2 implies that we can find such a set if $R_{U}^{b}<I(U ; Y)$. Since $(U, V)-X-Y$, by [7, Lemma 4]

$$
Z\left(\tilde{U}_{i} \mid \tilde{U}^{i-1}, X^{n}\right) \leq Z\left(\tilde{U}_{i} \mid \tilde{U}^{i-1}, Y^{n}\right),
$$

for all $i$, which implies that $Z\left(\tilde{U}_{i} \mid \tilde{U}^{i-1}, X^{n}\right) \leq \delta_{n}$ on $\mathcal{B}_{U}$ and $\mathcal{B}_{U} \subseteq \mathcal{A}_{U}$ for sufficiently large $n$. We also define two sets, the frozen set $\mathcal{F}_{U}$ and the deterministic set $\mathcal{D}_{U}$,

$$
\begin{aligned}
& \mathcal{F}_{U}=\left\{i \in \mathcal{A}_{U}^{c}: Z\left(\tilde{U}_{i} \mid \tilde{U}^{i-1}, X^{n}\right) \geq 1-\delta_{n}\right\}, \\
& \mathcal{D}_{U}=\left\{i \in \mathcal{A}_{U}^{c}: Z\left(\tilde{U}_{i} \mid \tilde{U}^{i-1}\right) \leq \delta_{n}\right\} .
\end{aligned}
$$

Note that $\mathcal{F}_{U}$ and $\mathcal{D}_{U}$ are disjoint for sufficiently large $n$. Similarly, we define $\mathcal{A}_{V}$ to be a subset of $[1: n]$ such that $\left|\mathcal{A}_{V}\right|=n R_{V}^{a}$, and

$$
Z\left(\tilde{V}_{i} \mid \tilde{V}^{i-1}, U^{n}, X^{n}\right) \geq 1-\delta_{n}, \text { or } Z\left(\tilde{V}_{i} \mid \tilde{V}^{i-1}, U^{n}\right) \leq \delta_{n},
$$

for all $i \in \mathcal{A}_{V}^{c}$. Hence $\mathcal{A}_{V}$ contains the set

$$
\begin{aligned}
&\left\{i: Z\left(\tilde{V}_{i} \mid \tilde{V}^{i-1}, U^{n}, X^{n}\right)\right.<1-\delta_{n}, \text { and } \\
&\left.Z\left(\tilde{V}_{i} \mid \tilde{V}^{i-1}, U^{n}\right)>\delta_{n}\right\} .
\end{aligned}
$$

Using the same reasoning, cf. Theorem 2theorem.3.2, the set $\mathcal{A}_{V}$ exists for sufficiently large $n$ if $R_{V}^{a}>I(X ; V \mid U)$. Let $\mathcal{B}_{V}$ be a subset of $[1: n]$ such that $\left|\mathcal{B}_{V}\right|=n R_{V}^{b}$ and

$$
\begin{aligned}
& \forall i \in \mathcal{B}_{V}: Z\left(\tilde{V}_{i} \mid \tilde{V}^{i-1}, U^{n}, Y^{n}\right) \leq \delta_{n}, \text { and } \\
& \qquad\left(\tilde{V}_{i} \mid \tilde{V}^{i-1}, U^{n}\right) \geq 1-\delta_{n} .
\end{aligned}
$$

The set $\mathcal{B}_{V}$ can be found if $R_{V}^{b}<I(V ; Y \mid U)$. Since $V-$ $(U, X)-(U, Y)$, we have again by [7, Lemma 4]

$$
Z\left(\tilde{V}_{i} \mid \tilde{V}^{i-1}, U^{n}, X^{n}\right) \leq Z\left(\tilde{V}_{i} \mid \tilde{V}^{i-1}, U^{n}, Y^{n}\right), i \in \mathcal{B}_{V},
$$

which implies that $\mathcal{B}_{V} \subseteq \mathcal{A}_{V}$ for sufficiently large $n$. We define two sets $\mathcal{F}_{V}$ and $\overline{\mathcal{D}}_{V}$ similarly as

$$
\begin{aligned}
& \mathcal{F}_{V}=\left\{i \in \mathcal{A}_{V}^{c}: Z\left(\tilde{V}_{i} \mid \tilde{V}^{i-1}, U^{n}, X^{n}\right) \geq 1-\delta_{n}\right\}, \\
& \mathcal{D}_{V}=\left\{i \in \mathcal{A}_{V}^{c}: Z\left(\tilde{V}_{i} \mid \tilde{V}^{i-1}, U^{n}\right) \leq \delta_{n}\right\} .
\end{aligned}
$$

As $n \rightarrow \infty, \mathcal{F}_{V}$ and $\mathcal{D}_{V}$ are eventually disjoint. In the following, we assume that $n$ is large enough so that $\mathcal{B}_{U} \subseteq \mathcal{A}_{U}$, $\mathcal{B}_{V} \subseteq \mathcal{A}_{V}$, and $\mathcal{F}_{U}$ and $\mathcal{D}_{U},\left(\mathcal{F}_{V}\right.$ and $\left.\mathcal{D}_{V}\right)$, are disjoint.

Encoding: We generate frozen symbols $\tilde{u}_{\mathcal{F}_{U}}$ and $\tilde{v}_{\mathcal{F}_{V}}$ i.i.d. with probability $1 / 2$ and reveal them to all parties. Given a source sequence $x^{n}$, a sequence $\tilde{u}^{n}$ is generated as

$$
\tilde{u}_{i}= \begin{cases}u & \text { with } p_{\tilde{U}_{i} \mid \tilde{U}^{i-1} X^{n}}\left(u \mid \tilde{u}^{i-1}, x^{n}\right) \text { if } i \in \mathcal{A}_{U} \\ u & \text { with } p_{\tilde{U}_{i} \mid \tilde{U}^{i-1}}\left(u \mid \tilde{u}^{i-1}\right) \text { if } i \in \mathcal{D}_{U}\end{cases}
$$

where $u \in\{0,1\}$. Then a sequence $\tilde{v}^{n}$ is chosen as

$$
\tilde{v}_{i}=\left\{\begin{array}{ll}
v & \text { with } p_{\tilde{V}_{i} \mid \tilde{V}^{i-1}} \tilde{U}^{n} X^{n}\left(v \mid \tilde{v}^{i-1}, \tilde{u}^{n}, x^{n}\right) \text { if } i \in \mathcal{A}_{V} \\
v & \text { with } p_{\tilde{V}_{i} \mid \tilde{V}^{i-1} \tilde{U}^{n}}\left(v \mid \tilde{v}^{i-1}, \tilde{u}^{n}\right) \text { if } i \in \mathcal{D}_{V}
\end{array},\right.
$$

where $v \in\{0,1\}$. We send coded symbols corresponding to the indices in sets $\hat{\mathcal{A}}_{U}=\mathcal{A}_{U} \backslash \mathcal{B}_{U}$ and $\hat{\mathcal{A}}_{V}=\mathcal{A}_{V} \backslash \mathcal{B}_{V}$ to the decoder. 
Decoding: Since the frozen symbols are shared between encoder and decoder we only need to retrieve the other indices. For $i \in \hat{\mathcal{A}}_{U}$, we set $\hat{u}_{i}=\tilde{u}_{i}$. When $i \in \mathcal{B}_{U}$, the symbol $\hat{u}_{i}$ is computed as

$$
\hat{u}_{i}=g_{i}^{Y}\left(\hat{u}^{i-1}, y^{n}\right)=\arg \max _{u} p_{\tilde{U}_{i} \mid \tilde{U}^{i-1} Y^{n}}\left(u \mid \hat{u}^{i-1}, y^{n}\right),
$$

and $\hat{u}_{i}$ is determined as

$$
\hat{u}_{i}=g_{i}\left(\hat{u}^{i-1}\right)=\arg \max _{u} p_{\tilde{U}_{i} \mid \tilde{U}^{i-1}}\left(u \mid \hat{u}^{i-1}\right),
$$

when $i \in \mathcal{D}_{U}$. We note that ties are broken arbitrarily.

Similarly, for $i \in \hat{\mathcal{A}}_{V}$ we set $\hat{v}_{i}=\tilde{v}_{i}$. In the index set $\mathcal{B}_{V}$ the $i$-th code symbol is recovered as

$$
\begin{aligned}
\hat{v}_{i} & =h_{i}^{Y}\left(\hat{v}^{i-1}, \hat{u}^{n}, y^{n}\right) \\
& =\arg \max _{v} p_{\tilde{V}_{i} \mid \tilde{V}^{i-1} \tilde{U}^{n} Y^{n}}\left(v \mid \hat{v}^{i-1}, \hat{u}^{n}, y^{n}\right),
\end{aligned}
$$

while for $i \in \mathcal{D}_{V}$

$$
\hat{v}_{i}=h_{i}\left(\hat{v}^{i-1}, \hat{u}^{n}\right)=\arg \max _{v} p_{\tilde{V}_{i} \mid \tilde{V}^{i-1} \tilde{U}^{n}}\left(v \mid \hat{v}^{i-1}, \hat{u}^{n}\right) .
$$

Ties are also broken arbitrarily. The decoder then outputs the sequence $\hat{x}^{n}$ symbol-wisely via $\hat{x}_{i}=f\left(\left(\hat{v}^{n} G_{n}\right)_{i}, y_{i}\right)$ for all $i \in[1: n]$.

Analysis: Since the frozen symbols are generated randomly, our aim is to prove that there exists a set of frozen symbols such that the distortion and equivocation levels are close to the desired values. Taking the randomness of frozen symbols into account, the encoding process induces the following probability distribution

$$
\begin{gathered}
\mathbb{Q}=p_{X^{n} Y^{n} E^{n}} \prod_{i \in \mathcal{A}_{U}} p\left(\tilde{u}_{i} \mid \tilde{u}^{i-1}, x^{n}\right) 2^{-\left|\mathcal{F}_{U}\right|} \prod_{i \in \mathcal{D}_{U}} p\left(\tilde{u}_{i} \mid \tilde{u}^{i-1}\right) \\
\prod_{i \in \mathcal{A}_{V}} p\left(\tilde{v}_{i} \mid \tilde{v}^{i-1}, \tilde{u}^{n}, x^{n}\right) 2^{-\left|\mathcal{F}_{V}\right|} \prod_{i \in \mathcal{D}_{V}} p\left(\tilde{v}_{i} \mid \tilde{v}^{i-1}, \tilde{u}^{n}\right) .
\end{gathered}
$$

Given the frozen symbols, message and side information, the decoding induces the conditional probability distribution

$$
\begin{aligned}
& \hat{\mathbb{Q}}=\prod_{\substack{i \in \hat{\mathcal{A}}_{U} \cup \mathcal{F}_{U}\\
}} \mathbf{1}\left\{\hat{u}_{i}=\tilde{u}_{i}\right\} \prod_{i \in \mathcal{B}_{U}} \mathbf{1}\left\{\hat{u}_{i}=g_{i}^{Y}\left(\hat{u}^{i-1}, y^{n}\right)\right\} \\
& \prod_{i \in \mathcal{D}_{U}} \mathbf{1}\left\{\hat{u}_{i}=g_{i}\left(\hat{u}^{i-1}\right)\right\} \prod_{i \in \mathcal{B}_{V}} \mathbf{1}\left\{\hat{v}_{i}=h_{i}^{Y}\left(\hat{v}^{i-1}, \hat{u}^{n}, y^{n}\right)\right\} \\
& \prod_{i \in \hat{\mathcal{A}}_{V} \cup \mathcal{F}_{V}} \mathbf{1}\left\{\hat{v}_{i}=\tilde{v}_{i}\right\} \prod_{i \in \mathcal{D}_{V}} \mathbf{1}\left\{\hat{v}_{i}=h_{i}\left(\hat{v}^{i-1}, \hat{u}^{n}\right)\right\} .
\end{aligned}
$$

Then the probability distribution induced by the whole process is given by

$$
\hat{\mathbb{P}}=\mathbb{Q} \times \hat{\mathbb{Q}} .
$$

We first examine the total variation distance between $\mathbb{P}$ and $\mathbb{Q}$. Pinsker's inequality [15, Lemma 11.6 .1$]$ tells us that

$$
\|\mathbb{P}-\mathbb{Q}\|_{T V} \leq \sqrt{2 \ln 2 D(\mathbb{P} \| \mathbb{Q})} .
$$

Then, by applying the chain rule of divergence and the Markov chain we obtain

$$
\begin{aligned}
& D(\mathbb{P} \| \mathbb{Q})=D\left(\mathbb{P}_{X^{n} Y^{n} E^{n}} \| \mathbb{Q}_{X^{n} Y^{n} E^{n}}\right) \\
& +\sum_{i=1}^{n} D\left(\mathbb{P}_{\tilde{U}_{i} \mid \tilde{U}^{i-1} X^{n}} \| \mathbb{Q}_{\tilde{U}_{i} \mid \tilde{U}^{i-1} X^{n}}\right)
\end{aligned}
$$

$$
\begin{aligned}
& +\sum_{i=1}^{n} D\left(\mathbb{P}_{\tilde{V}_{i} \mid \tilde{V}^{i-1} \tilde{U}^{n} X^{n}} \| \mathbb{Q}_{\tilde{V}_{i} \mid \tilde{V}^{i-1} \tilde{U}^{n} X^{n}}\right) \\
& \stackrel{(a)}{=} \sum_{i \in \mathcal{F}_{U}}\left(1-H\left(\tilde{U}_{i} \mid \tilde{U}^{i-1}, X^{n}\right)\right) \\
& +\sum_{i \in \mathcal{D}_{U}}\left(H\left(\tilde{U}_{i} \mid \tilde{U}^{i-1}\right)-H\left(\tilde{U}_{i} \mid \tilde{U}^{i-1}, X^{n}\right)\right) \\
& +\sum_{i \in \mathcal{F}_{V}}\left(1-H\left(\tilde{V}_{i} \mid \tilde{V}^{i-1}, \tilde{U}^{n}, X^{n}\right)\right) \\
& +\sum_{i \in \mathcal{D}_{V}}\left(H\left(\tilde{V}_{i} \mid \tilde{V}^{i-1}, \tilde{U}^{n}\right)-H\left(\tilde{V}_{i} \mid \tilde{V}^{i-1}, \tilde{U}^{n}, X^{n}\right)\right) \\
& \stackrel{(b)}{\leq} 2 \delta_{n}\left|\mathcal{F}_{U}\right|+\log _{2}(e) \delta_{n}\left|\mathcal{D}_{U}\right| \\
& +\sum_{i \in \mathcal{F}_{V}}\left(1-H\left(\tilde{V}_{i} \mid \tilde{V}^{i-1}, U^{n}, X^{n}\right)\right) \\
& +\sum_{i \in \mathcal{D}_{V}}\left(H\left(\tilde{V}_{i} \mid \tilde{V}^{i-1}, U^{n}\right)-H\left(\tilde{V}_{i} \mid \tilde{V}^{i-1}, U^{n}, X^{n}\right)\right) \\
& \leq \log _{2}(e) \delta_{n}\left(\left|\mathcal{D}_{U}\right|+\left|\mathcal{D}_{V}\right|\right)+2 \delta_{n}\left(\left|\mathcal{F}_{U}\right|+\left|\mathcal{F}_{V}\right|\right) \\
& =\mathcal{O}\left(2^{-n^{\beta^{\prime}}}\right),
\end{aligned}
$$

where $\beta^{\prime}<\beta$.

In (a) we have used the fact that for $i \in \mathcal{A}_{U}$, $\mathbb{Q}_{\tilde{U}_{i} \mid \tilde{U}^{i-1} X^{n}}=\mathbb{P}_{\tilde{U}_{i} \mid \tilde{U}^{i-1} X^{n}}$, while for $i \in \mathcal{F}_{U}, \mathbb{Q}_{\tilde{U}_{i} \mid \tilde{U}^{i-1} X^{n}}=$ $1 / 2$, and $\mathbb{Q}_{\tilde{U}_{i} \mid \tilde{U}^{i-1} X^{n}}=\mathbb{P}_{\tilde{U}_{i} \mid \tilde{U}^{i-1}}$ if $i \in \mathcal{D}_{U}$.

A similar reasoning is applied for the summation over sets related to $\tilde{V}^{n}$. The bijective correspondence between $U^{n}$ and $\tilde{U}^{n}$ is used in $(b)$, i.e., $H\left(\tilde{V}_{i} \mid \tilde{V}^{i-1}, \tilde{U}^{n}\right)=H\left(\tilde{V}_{i} \mid \tilde{V}^{i-1}, U^{n}\right)$, and similarly for the other terms. Additionally, for $i \in \mathcal{F}_{U}$ we have $Z\left(\tilde{U}_{i} \mid \tilde{U}^{i-1}, X^{n}\right) \geq 1-\delta_{n}$ which implies that (cf. (3Proofequation.3.3))

$$
\begin{aligned}
1-H\left(\tilde{U}_{i} \mid \tilde{U}^{i-1}, X^{n}\right) & \leq 1-\left(Z\left(\tilde{U}_{i} \mid \tilde{U}^{i-1}, X^{n}\right)\right)^{2} \\
& \leq 2 \delta_{n}-\delta_{n}^{2} \leq 2 \delta_{n} .
\end{aligned}
$$

A similar line of reasoning is applied when $i \in \mathcal{F}_{V}$. The inequality $Z\left(\tilde{U}_{i} \mid \tilde{U}^{i-1}\right) \leq \delta_{n}$ holds when $i \in \mathcal{D}_{U}$, which implies that (cf. (4Proofequation.3.4))

$$
H\left(\tilde{U}_{i} \mid \tilde{U}^{i-1}\right)-H\left(\tilde{U}_{i} \mid \tilde{U}^{i-1}, X^{n}\right) \leq \log _{2}(e) \delta_{n},
$$

for all $i \in \mathcal{D}_{U}$. Similar bounds are obtained for $i \in \mathcal{D}_{V}$. In summary (7Proofequation.3.7) implies that

$$
\|\mathbb{P}-\mathbb{Q}\|_{T V} \leq \mathcal{O}\left(2^{-n^{\gamma}}\right),
$$

where $\gamma<\beta^{\prime}$. As the next step, we bound the probability of error in the decoding. The error event is summarized as

$$
\begin{aligned}
\mathcal{E} & =\left\{\hat{U}^{n} \neq \tilde{U}^{n}, \text { or } \hat{V}^{n} \neq \tilde{V}^{n}\right\} \\
& \subseteq \mathcal{E}^{\prime}=\bigcup_{i \in \mathcal{B}_{U}} \mathcal{E}_{U}^{1 i} \bigcup_{i \in \mathcal{D}_{U}} \mathcal{E}_{U}^{2 i} \bigcup_{i \in \mathcal{B}_{V}} \mathcal{E}_{V}^{1 i} \bigcup_{i \in \mathcal{D}_{V}} \mathcal{E}_{V}^{2 i},
\end{aligned}
$$

due to the way that ties are resolved, where

$$
\begin{aligned}
& \mathcal{E}_{U}^{1 i}=\left\{\left(\tilde{u}^{n}, y^{n}\right): p\left(\tilde{u}_{i} \mid \tilde{u}^{i-1}, y^{n}\right) \leq p\left(\tilde{u}_{i} \oplus 1 \mid \tilde{u}^{i-1}, y^{n}\right)\right\} \\
& \mathcal{E}_{U}^{2 i}=\left\{\tilde{u}^{n}: p\left(\tilde{u}_{i} \mid \tilde{u}^{i-1}\right) \leq p\left(\tilde{u}_{i} \oplus 1 \mid \tilde{u}^{i-1}\right)\right\} \\
& \mathcal{E}_{V}^{1 i}=\left\{\left(\tilde{u}^{n}, \tilde{v}^{n}, y^{n}\right): p\left(\tilde{v}_{i} \mid \tilde{v}^{i-1}, \tilde{u}^{n}, y^{n}\right)\right. \\
& \left.\quad \leq p\left(\tilde{v}_{i} \oplus 1 \mid \tilde{v}^{i-1}, \tilde{u}^{n}, y^{n}\right)\right\} \\
& \mathcal{E}_{V}^{2 i}=\left\{\left(\tilde{u}^{n}, \tilde{v}^{n}\right): p\left(\tilde{v}_{i} \mid \tilde{v}^{i-1}, \tilde{u}^{n}\right) \leq p\left(\tilde{v}_{i} \oplus 1 \mid \tilde{v}^{i-1}, \tilde{u}^{n}\right)\right\} .
\end{aligned}
$$


From the definition of the total variation distance, i.e.,

$$
\|P-Q\|_{T V}=\sup _{\mathcal{E}}|P(\mathcal{E})-Q(\mathcal{E})|,
$$

and the union bound we obtain

$$
\begin{aligned}
\hat{\mathbb{P}}(\mathcal{E}) \leq \hat{\mathbb{P}}\left(\mathcal{E}^{\prime}\right) & =\mathbb{Q}\left(\mathcal{E}^{\prime}\right) \\
& \leq\|\mathbb{P}-\mathbb{Q}\|_{T V}+\mathbb{P}\left(\mathcal{E}^{\prime}\right) \\
& \leq\|\mathbb{P}-\mathbb{Q}\|_{T V}+\sum_{i \in \mathcal{B}_{U}} \mathbb{P}\left(\mathcal{E}_{U}^{1 i}\right)+\sum_{i \in \mathcal{D}_{U}} \mathbb{P}\left(\mathcal{E}_{U}^{2 i}\right) \\
& +\sum_{i \in \mathcal{B}_{V}} \mathbb{P}\left(\mathcal{E}_{V}^{1 i}\right)+\sum_{i \in \mathcal{D}_{V}} \mathbb{P}\left(\mathcal{E}_{V}^{2 i}\right) .
\end{aligned}
$$

For each $i \in \mathcal{B}_{U}$, the probability of the error event $\mathcal{E}_{U}^{1 i}$ is bounded by

$$
\begin{aligned}
& \mathbb{P}\left(\mathcal{E}_{U}^{1 i}\right) \\
& =\sum_{\tilde{u}^{i}, y^{n}} p\left(\tilde{u}^{i}, y^{n}\right) \mathbf{1}\left\{p\left(\tilde{u}_{i} \mid \tilde{u}^{i-1}, y^{n}\right) \leq p\left(\tilde{u}_{i} \oplus 1 \mid \tilde{u}^{i-1}, y^{n}\right)\right\} \\
& \leq \sum_{\tilde{u}^{i}, y^{n}} p\left(\tilde{u}^{i-1}, y^{n}\right) \sqrt{p\left(\tilde{u}_{i} \mid \tilde{u}^{i-1}, y^{n}\right) p\left(\tilde{u}_{i} \oplus 1 \mid \tilde{u}^{i-1}, y^{n}\right)} \\
& =Z\left(\tilde{U}_{i} \mid \tilde{U}^{i-1}, Y^{n}\right) \leq \delta_{n} .
\end{aligned}
$$

Similarly we obtain the following upper bound for each event $\mathcal{E}_{V}^{1 i}$, where $i \in \mathcal{B}_{V}$,

$$
\begin{aligned}
& \mathbb{P}\left(\mathcal{E}_{V}^{1 i}\right)=\sum_{\tilde{v}^{i}, \tilde{u}^{n}, y^{n}} p\left(\tilde{v}^{i}, \tilde{u}^{n}, y^{n}\right) \mathbf{1}\left\{p\left(\tilde{v}_{i} \mid \tilde{v}^{i-1}, \tilde{u}^{n}, y^{n}\right)\right. \\
& \leq \sum_{\tilde{v}^{i}, \tilde{u}^{n}, y^{n}} p\left(\tilde{v}^{i-1}, \tilde{u}^{n}, y^{n}\right) \sqrt{p\left(\tilde{v}_{i} \oplus 1 \mid \tilde{v}^{i-1}, \tilde{v}^{i-1}, \tilde{u}^{n}, y^{n}\right)} \\
& =\sum_{\left.\left.\tilde{v}^{i}, u^{n}, y^{n}\right)\right\}} p\left(\tilde{v}^{i-1}, u^{n}, y^{n}\right) \sqrt{p\left(\tilde{v}_{i} \mid \tilde{v}^{i-1}, u^{n}, y^{n}\right)} \\
& =Z\left(\tilde{V}_{i} \mid \tilde{V}^{i-1}, U^{n}, Y^{n}\right) \leq \delta_{n} .
\end{aligned}
$$

Using the same arguments the following bounds are valid for each term in the second and the fourth summations

$$
\begin{aligned}
& \mathbb{P}\left(\mathcal{E}_{U}^{2 i}\right) \leq Z\left(\tilde{U}_{i} \mid \tilde{U}^{i-1}\right) \leq \delta_{n}, \quad \forall i \in \mathcal{D}_{U}, \\
& \mathbb{P}\left(\mathcal{E}_{V}^{2 i}\right) \leq Z\left(\tilde{V}_{i} \mid \tilde{V}^{i-1}, U^{n}\right) \leq \delta_{n}, \quad \forall i \in \mathcal{D}_{V} .
\end{aligned}
$$

Hence in summary the probability of error under the distribution induced by the encoding and decoding process is bounded by

$$
\hat{\mathbb{P}}(\mathcal{E})=\mathcal{O}\left(2^{-n^{\beta^{\prime}}}\right) .
$$

With all available components, define the sequence $\tilde{x}^{n}$ as $\tilde{x}_{i}=f\left(\left(\tilde{v}^{n} G_{n}\right)_{i}, y_{i}\right)=f\left(v_{i}, y_{i}\right)$. Since the distortion measure is a bounded mapping, by the law of total expectation, the distortion at the destination is upper bounded as

$$
\begin{aligned}
\mathbb{E}_{\hat{\mathbb{P}}}\left[d\left(X^{n}, \hat{X}^{n}\right)\right]=\hat{\mathbb{P}}\left(\mathcal{E}^{c}\right) \mathbb{E}_{\hat{\mathbb{P}}}\left[d\left(X^{n}, \hat{X}^{n}\right) \mid \mathcal{E}^{c}\right] \\
\quad+\hat{\mathbb{P}}(\mathcal{E}) \mathbb{E}_{\hat{\mathbb{P}}}\left[d\left(X^{n}, \hat{X}^{n}\right) \mid \mathcal{E}\right] \\
\quad \leq \hat{\mathbb{P}}\left(\mathcal{E}^{c}\right) \mathbb{E}_{\hat{\mathbb{P}}}\left[d\left(X^{n}, \hat{X}^{n}\right) \mid \mathcal{E}^{c}\right]+d_{\max } \hat{\mathbb{P}}(\mathcal{E}) .
\end{aligned}
$$

We observe that given $\mathcal{E}^{c}, \tilde{u}_{i}=g_{i}^{Y}\left(\tilde{u}^{i-1}, y^{n}\right)$ for all $i \in \mathcal{B}_{U}$ and so on. Hence for consistent $\left(\tilde{u}^{n}, \tilde{v}^{n}\right) \in \mathcal{E}^{c}$, we have

$$
\begin{aligned}
& \hat{\mathbb{P}}\left(x^{n}, y^{n}, e^{n}, \tilde{u}^{n}, \hat{u}^{n}, \tilde{v}^{n}, \hat{v}^{n} \mid \mathcal{E}^{c}\right) \\
& =\frac{1}{\hat{\mathbb{P}}\left(\mathcal{E}^{c}\right)} \mathbb{Q}\left(x^{n}, y^{n}, e^{n}, \tilde{u}^{n}, \tilde{v}^{n}\right) \times \mathbf{1}\left\{\tilde{u}^{n}=\hat{u}^{n}, \tilde{v}^{n}=\hat{v}^{n}\right\} .
\end{aligned}
$$

Using a property of the total variation distance (cf. [16, Property $2 \mathrm{~b}]$ ), the distortion is further bounded as

$$
\begin{aligned}
\mathbb{E}_{\hat{\mathbb{P}}}\left[d\left(X^{n}, \hat{X}^{n}\right)\right] \leq \sum_{x^{n}, \tilde{v}^{n} \in \mathcal{E}^{c}} Q\left(x^{n}, \tilde{v}^{n}\right) d\left(x^{n}, \tilde{x}^{n}\right)+d_{\max } \hat{\mathbb{P}}(\mathcal{E}) \\
\quad \leq \mathbb{E}_{\mathbb{Q}}\left[d\left(X^{n}, \tilde{X}^{n}\right)\right]+d_{\max } \hat{\mathbb{P}}(\mathcal{E}) \\
\leq \mathbb{E}_{\mathbb{P}}\left[d\left(X^{n}, \tilde{X}^{n}\right)\right]+d_{\max }\left(\|\mathbb{P}-\mathbb{Q}\|_{T V}+\hat{\mathbb{P}}(\mathcal{E})\right) \\
\leq D+\mathcal{O}\left(2^{-n^{\beta^{\prime}}}\right) .
\end{aligned}
$$

Since the frozen symbols are also available at the eavesdropper, the equivocation level is calculated as

$$
\begin{aligned}
& H_{\hat{\mathbb{P}}}\left(X^{n} \mid \tilde{U}_{\hat{\mathcal{A}}_{U} \cup \mathcal{F}_{U}}, \tilde{V}_{\hat{\mathcal{A}}_{V} \cup \mathcal{F}_{V}}, E^{n}\right) \\
& =H_{\mathbb{Q}}\left(X^{n} \mid \tilde{U}_{\hat{\mathcal{A}}_{U} \cup \mathcal{F}_{U}}, \tilde{V}_{\hat{\mathcal{A}}_{V} \cup \mathcal{F}_{V}}, E^{n}\right) \\
& \geq H_{\mathbb{Q}}\left(X^{n} \mid \tilde{U}^{n}, E^{n}\right)-I_{\mathbb{Q}}\left(X^{n} ; \tilde{V}_{\hat{\mathcal{A}}_{V} \cup \mathcal{F}_{V}} \mid \tilde{U}^{n}, E^{n}\right) \\
& =H_{\mathbb{Q}}\left(X^{n} \mid \tilde{U}^{n}, E^{n}\right)-I_{\mathbb{Q}}\left(X^{n} ; \tilde{V}_{\hat{\mathcal{A}}_{V}} \mid \tilde{V}_{\mathcal{F}_{V}}, \tilde{U}^{n}, E^{n}\right),
\end{aligned}
$$

as under $\mathbb{Q}, \tilde{V}_{\mathcal{F}_{V}}$ is independent of $\left(X^{n}, E^{n}, \tilde{U}^{n}\right)$. Since

$$
H_{\mathbb{Q}}\left(\tilde{V}_{\hat{\mathcal{A}}_{V}}\right) \leq n\left(R_{V}^{a}-R_{V}^{b}\right),
$$

the equivocation is lower bounded by

$$
\begin{aligned}
& \frac{1}{n} H_{\hat{\mathbb{P}}}\left(X^{n} \mid \tilde{U}_{\hat{\mathcal{A}}_{U} \cup \mathcal{F}_{U}}, \tilde{V}_{\hat{\mathcal{A}}_{V} \cup \mathcal{F}_{V}}, E^{n}\right) \\
& \quad \geq \frac{1}{n} H_{\mathbb{Q}}\left(X^{n} \mid \tilde{U}^{n}, E^{n}\right)-\left(R_{V}^{a}-R_{V}^{b}\right) .
\end{aligned}
$$

Since $\|\mathbb{P}-\mathbb{Q}\|_{T V}=\mathcal{O}\left(2^{-n^{\beta^{\prime}}}\right)$, applying [15, Theorem 17.3.3] yields

$$
\frac{1}{n}\left|H_{\mathbb{P}}\left(X^{n} \mid \tilde{U}^{n}, E^{n}\right)-H_{\mathbb{Q}}\left(X^{n} \mid \tilde{U}^{n}, E^{n}\right)\right|=\mathcal{O}\left(2^{-n^{\beta^{\prime}}}\right) .
$$

Therefore we obtain

$$
\begin{aligned}
& \underbrace{\frac{1}{n} H_{\hat{\mathbb{P}}}\left(X^{n} \mid \tilde{U}_{\hat{\mathcal{A}}_{U} \cup \mathcal{F}_{U}}, \tilde{V}_{\hat{\mathcal{A}}_{V} \cup \mathcal{F}_{V}}, E^{n}\right)}_{\Delta_{L}} \\
& \quad \geq \underbrace{H(X \mid U, E)-\left(R_{V}^{a}-R_{V}^{b}\right)-\mathcal{O}\left(2^{-n^{\beta^{\prime}}}\right)}_{\Delta_{R}} .
\end{aligned}
$$

Having calculated average distortion and equivocation levels over randomly chosen frozen symbols, we proceed to show that there exists a set of frozen symbols $\tilde{u}_{\mathcal{F}_{U}}$ and $\tilde{v}_{\mathcal{F}_{V}}$ such that the desired distortion and equivocation levels are achievable. Define random variables $T$ and $S$ as

$$
\begin{aligned}
& T\left(\tilde{u}_{\mathcal{F}_{U}}, \tilde{v}_{\mathcal{F}_{V}}\right)=\mathbb{E}_{\hat{\mathbb{P}}}\left[d\left(X^{n}, \hat{X}^{n}\right) \mid \tilde{u}_{\mathcal{F}_{U}}, \tilde{v}_{\mathcal{F}_{V}}\right], \\
& S\left(\tilde{u}_{\mathcal{F}_{U}}, \tilde{v}_{\mathcal{F}_{V}}\right)=\frac{1}{n} H_{\hat{\mathbb{P}}}\left(X^{n} \mid \tilde{U}_{\hat{\mathcal{A}}}, \tilde{u}_{\mathcal{F}_{U}}, \tilde{V}_{\hat{\mathcal{A}}_{V}}, \tilde{v}_{\mathcal{F}_{V}}, E^{n}\right) .
\end{aligned}
$$

We construct a super code by concatenating $N$ identical copies of our construction, in which the random choice of frozen 
symbols is mutually independent. Hence the distortion and equivocation of the resulting code are given by

$$
\frac{1}{N} \sum_{j=1}^{N} T_{j}, \text { and } \frac{1}{N} \sum_{j=1}^{N} S_{j},
$$

where $T_{i}$ are i.i.d. copy of $T$ and similarly for $S_{i}$. For any given $\epsilon>0$ as $N \rightarrow \infty$, by weak law of large numbers, we have

$$
\begin{array}{r}
\operatorname{Pr}\left\{\left|\frac{1}{N} \sum_{j=1}^{N} T_{j}-\mathbb{E}_{\hat{\mathbb{P}}}\left[d\left(X^{n}, \hat{X}^{n}\right)\right]\right|<\epsilon\right\} \rightarrow 1, \\
\operatorname{Pr}\left\{\left|\frac{1}{N} \sum_{j=1}^{N} S_{j}-\Delta_{L}\right|<\epsilon\right\} \rightarrow 1 .
\end{array}
$$

Therefore, for sufficiently large $N$,

$$
\operatorname{Pr}\left\{\frac{1}{N} \sum_{j=1}^{N} T_{j}<D+\mathcal{O}\left(2^{-n^{\beta^{\prime}}}\right), \frac{1}{n} \sum_{j=1}^{N} S_{j}>\Delta_{R}\right\}>0,
$$

which implies there exists a set frozen symbols of a super code such that the distortion $D$ and equivocation $H(X \mid U, E)-$ $\left(R_{V}^{a}-R_{V}^{b}\right)$ are achievable. Using Fourier-Motzkin elimination method we obtain

$$
\begin{aligned}
& R \geq I(X ; V \mid Y), \\
& \Delta \leq H(X \mid U, E)-I(X ; V \mid U, Y) .
\end{aligned}
$$

\section{CONCLUSION}

In this work, we reconsidered the problem of secure WynerZiv source coding with side information at the eavesdropper. We showed that the rate-distortion-equivocation region can be achieved using a polar coding scheme. Our result provides the basis for further developments to an efficient, secure compressing method for future big data applications.

\section{REFERENCES}

[1] D. Gunduz, E. Erkip, and H. V. Poor, "Secure lossless compression with side information," in Information Theory Workshop, 2008. ITW'08. IEEE. IEEE, 2008, pp. 169-173.

[2] J. Villard and P. Piantanida, "Secure multiterminal source coding with side information at the eavesdropper," IEEE Trans. Inf. Theory, vol. 59, no. 6, pp. 3668-3692, 2013.

[3] E. Arikan, "Channel polarization: A method for constructing capacityachieving codes for symmetric binary-input memoryless channels," IEEE Trans. Inf. Theory, vol. 55, no. 7, pp. 3051-3073, 2009.

[4] R. Mori and T. Tanaka, "Channel polarization on q-ary discrete memoryless channels by arbitrary kernels," in 2010 IEEE International Symposium on Information Theory. IEEE, 2010, pp. 894-898.

[5] E. Sasoglu, E. Telatar, and E. Arıkan, "Polarization for arbitrary discrete memoryless channels," arXiv preprint arXiv:0908.0302, 2009.

[6] E. Arikan, "Source polarization," in IEEE International Symposium on Information Theory-Proceedings, pp. 899-903.

[7] N. Goela, E. Abbe, and M. Gastpar, "Polar codes for broadcast channels," IEEE Trans. Inf. Theory, vol. 61, no. 2, pp. 758-782, 2015.

[8] J. Honda and H. Yamamoto, "Polar coding without alphabet extension for asymmetric models," IEEE Trans. Inf. Theory, vol. 59, no. 12, pp. 7829-7838, 2013.

[9] M. Andersson, V. Rathi, R. Thobaben, J. Kliewer, and M. Skoglund, "Nested polar codes for wiretap and relay channels," IEEE Commun. Lett., vol. 14, no. 8, pp. 752-754, 2010.
[10] H. Mahdavifar and A. Vardy, "Achieving the secrecy capacity of wiretap channels using polar codes," IEEE Trans. Inf. Theory, vol. 57, no. 10, pp. 6428-6443, 2011.

[11] M. Andersson, R. F. Schaefer, T. J. Oechtering, and M. Skoglund, "Polar coding for bidirectional broadcast channels with common and confidential messages," IEEE Journal on Selected Areas in Communications, vol. 31, no. 9, pp. 1901-1908, 2013.

[12] F. Mokhtarinezhad, J. Kliewer, and O. Simeone, "Lossy compression with privacy constraints: Optimality of polar codes," in IEEE Information Theory Workshop-Fall (ITW),. IEEE, 2015, pp. 182-186.

[13] I. Tal and A. Vardy, "List decoding of polar codes," IEEE Trans. Inf. Theory, vol. 61, no. 5, pp. 2213-2226, 2015.

[14] A. Wyner and J. Ziv, "The rate-distortion function for source coding with side information at the decoder," IEEE Trans. Inf. Theory, vol. 22, no. 1 , pp. 1-10, 1976.

[15] T. M. Cover and J. A. Thomas, Elements of information theory. John Wiley \& Sons, 2012.

[16] C. Schieler and P. Cuff, "Rate-distortion theory for secrecy systems," IEEE Trans. Inf. Theory, vol. 60, no. 12, pp. 7584-7605, 2014. 